\title{
A Novel In Vitro Liver Cell Culture Flow System Allowing Long-Term Metabolism and Hepatotoxicity Studies
}

\author{
Stefanie Buesch, Jenny Schroeder, Maureen Bunger, Theresa D'Souza, and Magdalene Stosik
}

\begin{abstract}
Introduction: Hepatotoxicity is a concern when developing new pharmaceutical compounds. Animal models do not accurately predict toxicity in humans due to species differences, for example, in compound metabolism. In this study, we present an alternative in vitro model based on the culture of primary human hepatocytes (PHH) in the Quasi Vivo ${ }^{\circledR}$ QV900 Flow System.

Materials and Methods: PHH were kept in sandwich culture in static and in perfused conditions. During 3 weeks of culture, viability and albumin content were quantified. Basal and induced cytochrome P450 (CYP) enzyme activity were assessed by luminescence assay and analysis of metabolite formation.

Results: Good cell viability and sustained albumin production were observed in perfused culture. In comparison to nonperfused cultures, the basal activity of the drug metabolism enzymes CYP3A4, CYP1A2, and CYP2B6 was increased. Induced CYP3A4 activity was comparable between perfused and nonperfused cultures. Furthermore, the QV900 System allowed fewer medium changes as typically required in static cultures. This enables buildup of potential toxic metabolites that would otherwise not be detected when media are refreshed daily.

Discussion: PHH can be cultured in the QV900 Flow System for 3 weeks with only two medium changes per week. Improved basal CYP activity was observed in comparison to static culture.

Conclusion: We present a liver cell culture flow system that improves longer-term stability of drug metabolism enzymes in PHH and is therefore a more human-relevant in vitro model for repeat dosing of hepatotoxicants.
\end{abstract}

Keywords: cytochrome P450 enzyme, drug metabolism, hepatocyte, hepatotoxicity, liver

\section{Introduction}

$\mathbf{H}$ EPATOTOXICITY IS A CONCERN when developing new small molecule pharmaceutical compounds. One of the major reasons for drug withdrawal from market is hepatotoxicity ${ }^{1}$ that does not arise immediately, but requires patients to have prolonged and repeated exposure to drugs.

Animal models can be suitable for the detection of hepatotoxicity, including long-term and repeat dosing studies to replicate this long-term phenomenon. However, animal models often do not accurately predict toxicity in humans ${ }^{2,3}$ due to species differences, including differences in expression, structure, and catalytic activities of compound metabolizing enzymes. Furthermore, ethical concerns limit the implemen- tation of animals in toxicity studies. In vitro testing systems are gaining importance in the field of toxicity testing. ${ }^{4}$

Primary human hepatocytes $(\mathrm{PHH})$ are the most accurate model of human liver physiology 5,6 and pathology. While there is increased interest in using in vitro PHH to predict liver toxicity in humans, PHH cultures undergo rapid dedifferentiation ${ }^{7-9}$ and have short lifespans in standard culture systems, limiting their utility for long-term and delayedonset hepatotoxicity models.

Currently, the static sandwich culture consisting of primary hepatocytes growing between layers of collagen and/ or basement membrane extract represents the gold standard for PHH culture. ${ }^{10,11}$ While sandwich-based systems are known to exhibit prolonged viability ${ }^{10}$ and functionality in

\footnotetext{
${ }^{1}$ Lonza Cologne GmbH, Cologne, Germany.

${ }^{2}$ Lonza RTP, Inc., Research Triangle Park, North Carolina.

${ }^{3}$ Lonza Walkersville, Inc., Walkersville, Maryland.
}

(c) Stefanie Buesch et al., 2018; Published by Mary Ann Liebert, Inc. This Open Access article is distributed under the terms of the Creative Commons License (http://creativecommons.org/licenses/by/4.0), which permits unrestricted use, distribution, and reproduction in any medium, provided the original work is properly cited. 
terms of morphology, cytochrome P450 (CYP) enzyme activity, ${ }^{12}$ and bile canaliculi formation, ${ }^{13}$ they are limited in durations that these phenotypes are maintained to about 7 days in culture. In addition to de-differentiation, maintenance of $\mathrm{PHH}$ in culture is time-consuming, including frequent medium changes that prevent the accumulation of metabolites and other factors that may contribute to delayed-onset toxicity.

Therefore, there is a need for in vitro culture systems that stably maintain full metabolic activity of PHH over longer terms, ${ }^{14,15}$ thus allowing for development of human repeat dosing and long-term exposure models in vitro.

In previous research, fluid shear stress was demonstrated to influence hepatocyte viability, albumin and urea synthesis, ${ }^{16,17}$ and to modulate $C Y P$ gene expression ${ }^{18,19}$ over time.

In this study, we describe the application of fluid flow to 2D PHH sandwich culture using the Quasi Vivo ${ }^{\circledR}$ QV900 Flow System. The QV900 System consists of six chambers that can be connected in flexible configurations (Fig. 1). A peristaltic pump generates a cell culture medium flow that mimics laminar interstitial fluid flow and ensures a steady supply of nutrients, growth factors, and oxygen, as well as removal of waste products and continuous dosing with test compounds.

In contrast to the daily medium changes required for static sandwich culture, in the QV900 System, biweekly medium changes were sufficient to maintain good cell viability. In addition, increased basal activity of key metabolic enzymes was observed for up to 21 days in the QV900 System compared to static sandwich culture.

\section{Materials and Methods}

\section{Cells and media}

Cryopreserved Human Hepatocytes, Plateable, Induction Qualified (Lot HUM4152; Lonza), were cultured according to the supplier's instructions. Briefly, 400,000 cells were plated per one $12 \mathrm{~mm}$ collagen-coated coverslips (Neuvitro). Four to 6 hours postseeding, Matrigel ${ }^{\mathrm{TM}}$ (Corning) was added in Hepatocyte Culture Medium (HCM ${ }^{\mathrm{TM}}$; Lonza). Twentyfour hours after seeding, coverslips were transferred to Quasi Vivo QV900 trays (QV900; Lonza) or fresh 24-well plates, respectively. The medium was renewed daily (standard sandwich culture) or twice per week (QV900). Flow rate in the QV900 System was set to $\sim 130 \mu \mathrm{L} / \mathrm{min}$ using a dedicated Parker Hannifin Cell Culture Tray Pump PF600 Range (Lonza). Three chambers were connected in series to one pump head. Both static and perfused samples were cultured at $37^{\circ} \mathrm{C}, 5 \% \mathrm{CO}_{2}$, in a humidified incubator. For analysis of viability and albumin secretion, coverslips were transferred into fresh 24-well plates for 1.5 hours to ensure optimal comparability of the results.

Viability and metabolic capacity of the culture were assessed by CellTiter-Blue ${ }^{\circledR}$ Cell Viability Assay (Promega)

A
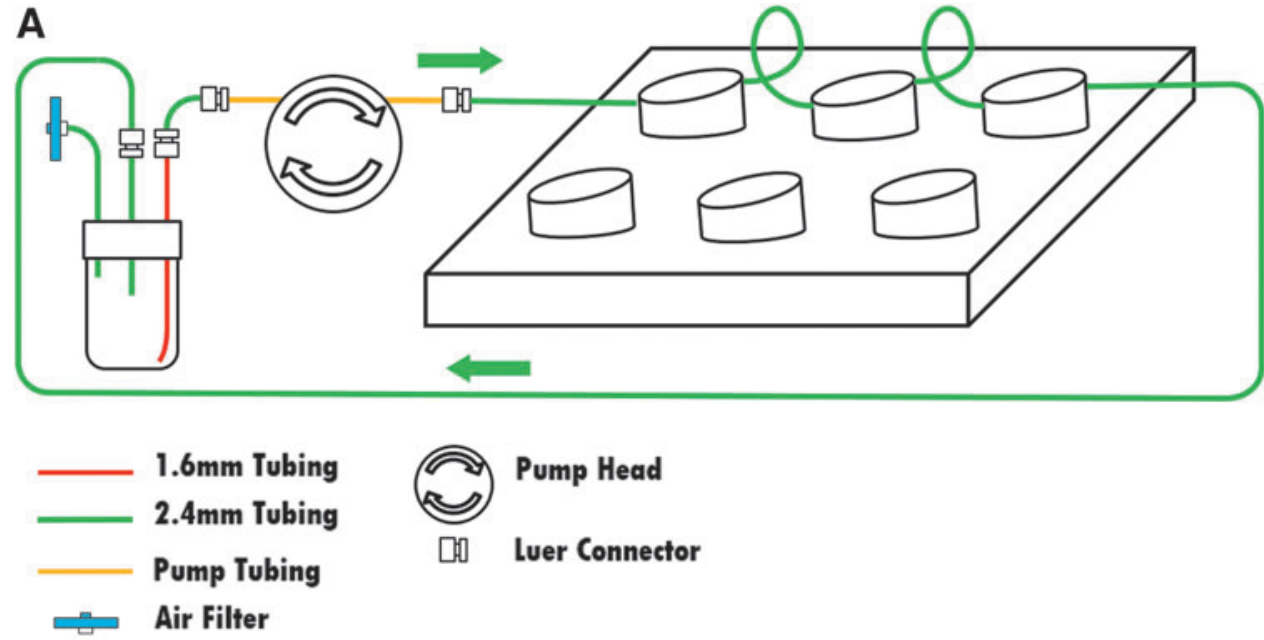

B 
according to the manufacturer's instructions. Albumin content in the supernatant was quantified with the Human Albumin ELISA kit (Bethyl) according to the manufacturer's instructions. Indirect measurement of CYP3A4 activity was assessed by the P450-Glo ${ }^{\mathrm{TM}}$ CYP3A4 Assay (Promega) using Luciferin-IPA as a substrate according to the manufacturer's instructions. For direct measurement of CYP3A4, CYP2B6, and CYP1A2 metabolic activity, PHH were incubated with $200 \mu \mathrm{M}$ testosterone, $250 \mu \mathrm{M}$ bupropion, and $100 \mu \mathrm{M}$ phenacetin for 15 minutes. Formation of $6 \beta$-hydroxytestosterone, $\mathrm{OH}$ bupropion, and acetaminophen was evaluated, as analyzed by liquid chromatography-tandem mass spectrometry (Biotranex). For measuring the inducibility of CYP3A4, PHH were incubated with $10 \mu \mathrm{M}$ rifampicin or dimethyl sulfoxide (DMSO) control for 72 hours before measuring the CYP3A4 activity by P450-Glo CYP3A4 Assay (Promega).

\section{Results}

In this study, we evaluated the Quasi Vivo QV900 Flow System (Fig. 1A, B) for improving the phenotype of $\mathrm{PHH}$ cultures. The QV900 was chosen for this study for two main reasons. First, unlike the silicone chambers used in previous research, ${ }^{19}$ the QV900 is made of an acrylate plastic that is designed to reduce nonspecific binding and therefore more amenable for long-term chemical and drug treatments. ${ }^{20}$ Second, the QV900 was designed in combination with a 6channel pump and tray system to provide a self-contained unit that can be easily transferred into and out of incubators, and is more suited for higher throughput applications with better statistical power.

Moreover, unlike previous studies, this study utilizes cryopreserved hepatocytes rather than freshly isolated hepatocytes. The cryopreservation process elicits greater stress on hepatocytes, leading to lower metabolic activities and reduced phenotypic stability upon thawing and plating. However, using cryopreserved hepatocytes is more favorable as the batch-to-batch variability of hepatocyte isolations is high and cryopreservation enables researchers to perform repeated testing on the same batch over time for better consistency and comparability across experiments and tests.

To test the capacity of the QV900 to maintain and even improve hepatocyte function over multiple weeks, cryopreserved human hepatocytes were cultured in the QV900 and compared to cryopreserved hepatocytes cultured in standard static sandwich culture.

We used a perfusion setup in which three wells of the QV900 were connected in series to one pump head (Fig. 1A). This allowed for the 72-hour rifampicin treatment of defined triplicate samples at specified time points without affecting untreated samples and DMSO-treated controls, making the most efficient use of the Parker pump equipped with six pump heads. Accordingly, 18 samples could be perfused in 1 experiment.

Viability was measured indirectly using a resazurin metabolism assay comparing between static and perfused cultures. As shown in Figure 2A, comparable resazurin metabolism was observed after 4 and 11 days of both static and perfused cultures, followed by a decline toward day 21 (Fig. 2A).

Another measure of hepatocyte cell health, albumin production (Fig. 2B), was sustained for 14 days in culture in both systems and was detectable during the complete culture period of 3 weeks. The amount of albumin secretion seems to correlate
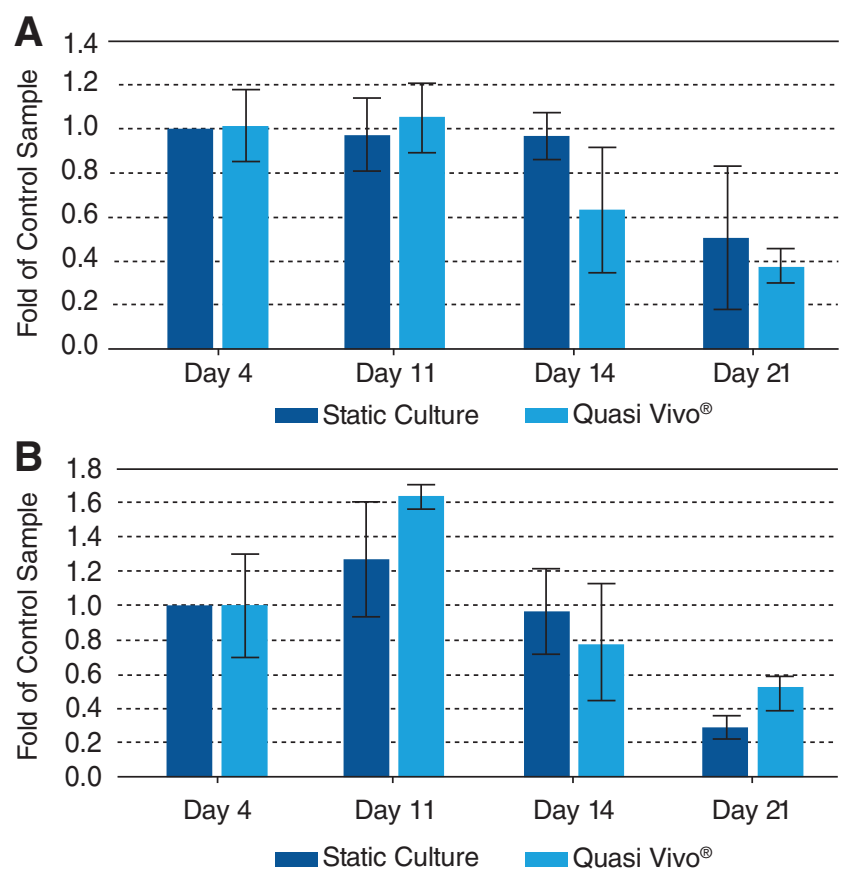

FIG. 2. Viability and albumin secretion of PHH are comparable in static and flow culture. PHH in collagen/Matrigel $^{\mathrm{TM}}$ sandwich culture were cultured for the indicated period of time in either static conditions or in Quasi Vivo QV900 Flow System. (A) Viability was assessed with the CellTiter-Blue ${ }^{\circledR}$ Cell Viability Assay (Promega). (B) Albumin content in the supernatant was quantified with the Human Albumin ELISA kit (Bethyl). Data of three independent experiments are shown, $n \geq 3$. Error bars indicate standard deviation. The control sample (day 4 standard culture) was set to 1 in every experiment for normalization. $\mathrm{PHH}$, primary human hepatocytes.

with the metabolic activity of cells as an indirect measure of cell number and viability. The typical cobblestone morphology of healthy hepatocytes was maintained throughout culture in both static and perfused cultures (Fig. 3).

Expression of drug-metabolizing enzymes is one key property of hepatocytes. In comparison to nonperfused cultures, the basal activity of the drug metabolism enzyme CYP3A4 on day 4, 14, and 21 was increased 2- to 13-fold as measured by the luciferase assay (Fig. 4A). CYP3A4 activity was continuously increasing during the incubation period of 3 weeks in QV900 flow culture, while remaining at low initial level in static culture. This finding was confirmed by analysis of the conversion of testosterone to $6 \beta$-hydroxytestosterone as a measure of CYP3A4 activity (Fig. 4B). In a similar way, the basal CYP1A2 activity was increased up to 10-fold in QV900 flow culture as determined by quantification of phenacetin metabolite, acetaminophen, on day 14 and 21 (Fig. 4D). Basal CYP2B6 activity was increasing on day 14 and sustained on day 21 of the QV900 flow culture, while it was remaining at initial level at day 14 and sinking even further at day 21 in static culture (Fig. 4C).

In addition to the basal CYP activity, the inducibility of such enzymes by certain reference compounds is an important parameter of hepatocyte functionality. Monitoring the change of CYP activity after treatment with known or new pharmaceutical compounds provides information about a risk for 

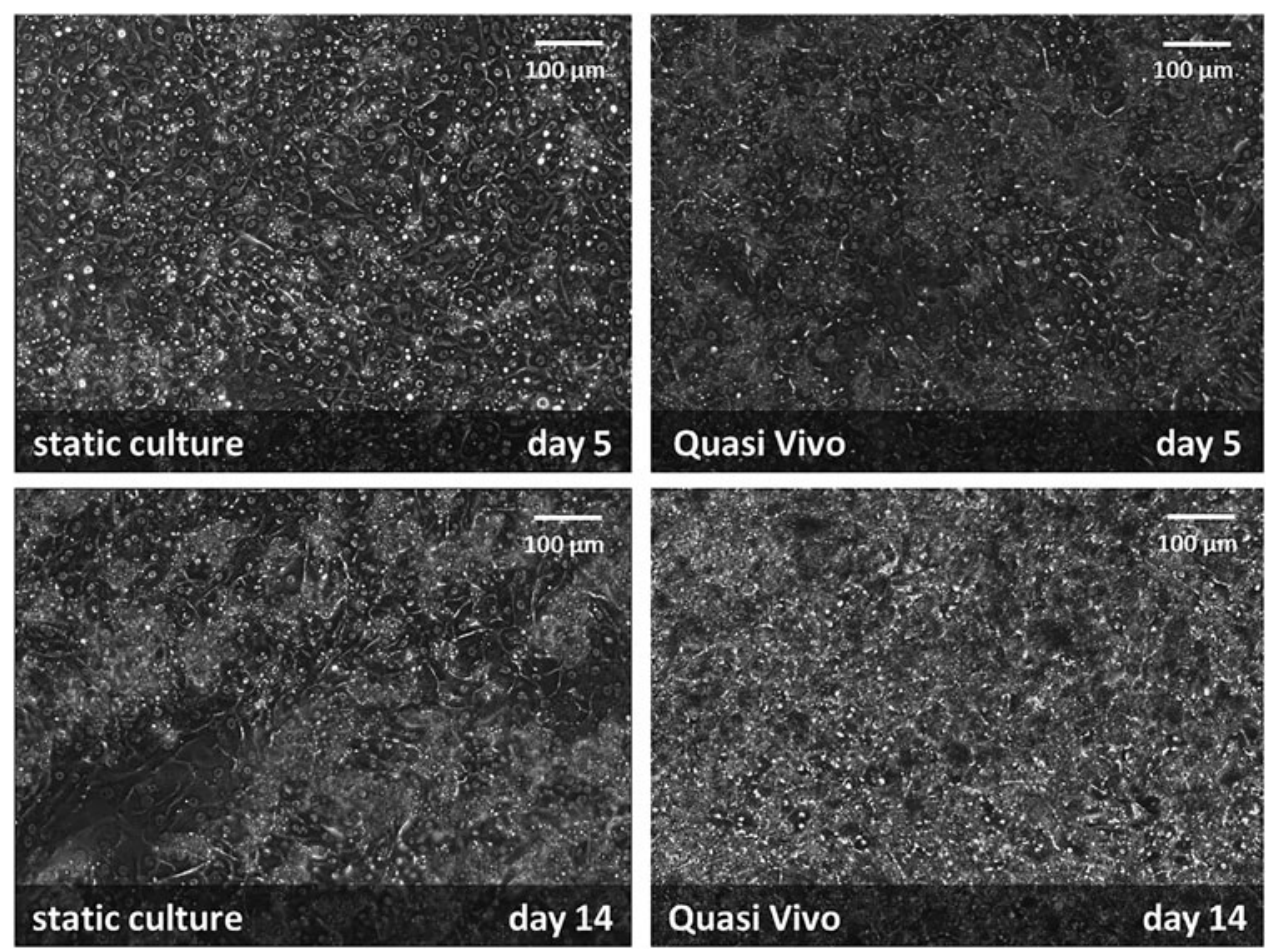

FIG. 3. The morphology of $\mathrm{PHH}$ is comparable in static and flow culture. PHH in collagen/Matrigel sandwich culture were cultured for the indicated period in either static culture conditions or in Quasi Vivo QV900 Flow System.
A

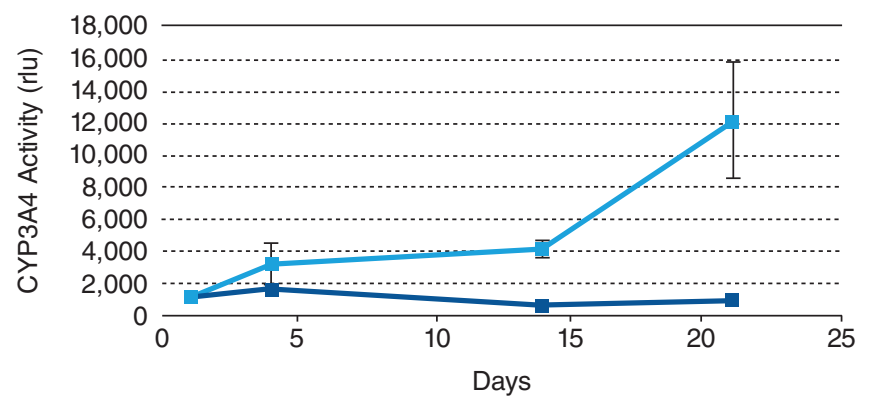

C

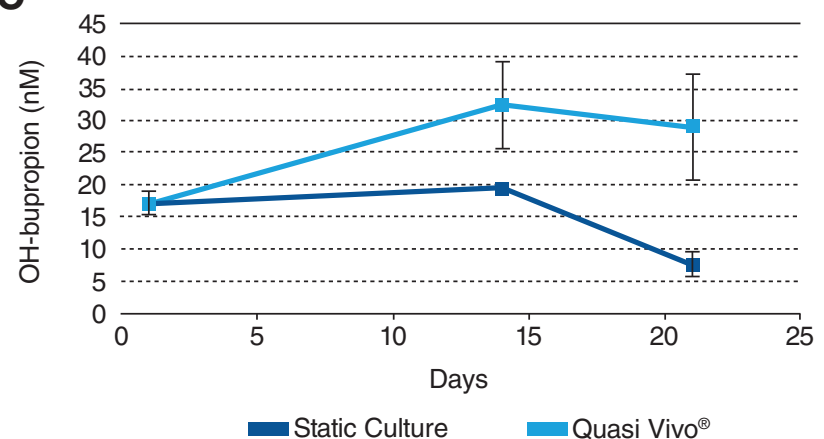

B
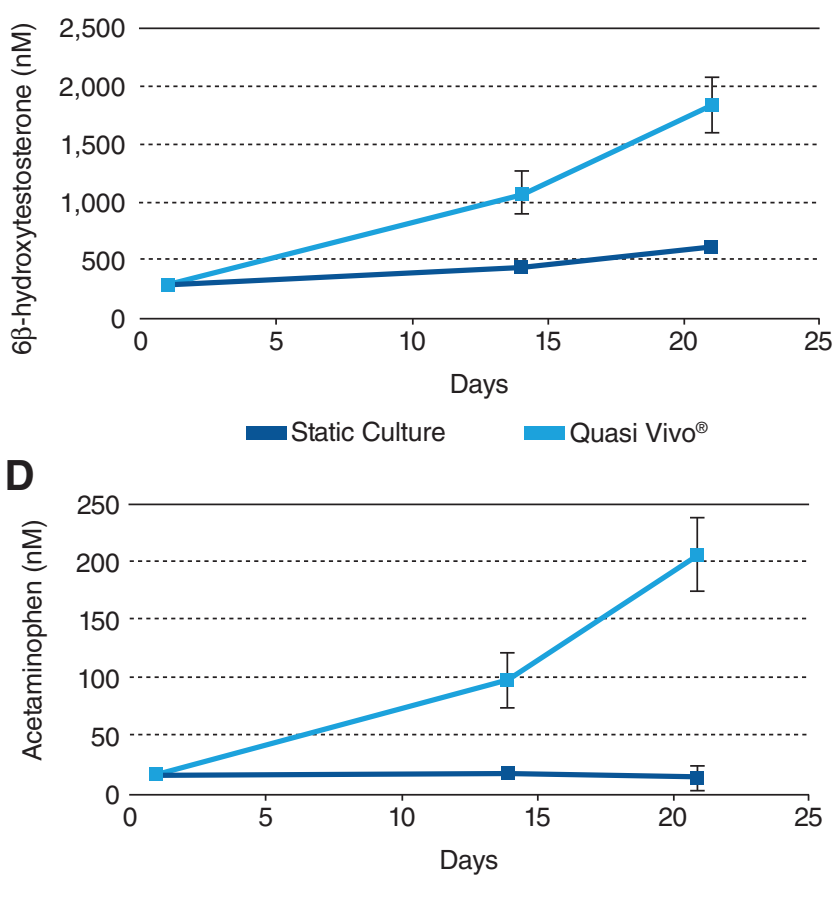

Static Culture $\quad$ Quasi Vivo ${ }^{\circledR}$

FIG. 4. The basal activity of CYP3A4, CYP2B6, and CYP1A2 is increased in PHH cultured in Quasi Vivo QV900 Flow System. PHH in collagen/Matrigel sandwich culture were cultured for the indicated period in either static conditions or in QV900 Flow System. (A) CYP3A4 activity was assessed with the P450-Glo ${ }^{\mathrm{TM}}$ CYP3A4 Assay (Promega) using LuciferinIPA as a substrate. Results of one typical experiment are shown, $n=3$. Error bars indicate standard deviation. (B) For measurement of CYP3A4 activity, cells were incubated with $200 \mu \mathrm{M}$ testosterone for 15 minutes and the formation of $6 \beta$-hydroxytestosterone was evaluated. (C) For measurement of CYP2B6 activity, PHH were incubated with $250 \mu$ M bupropion for 15 minutes and the formation of OH-bupropion was evaluated. (D) For measurement of CYP1A2 activity, PHH were incubated with $100 \mu \mathrm{M}$ phenacetin for 15 minutes and the formation of acetaminophen was evaluated. All culture supernatants were analyzed at Biotranex LLC by liquid chromatography-tandem mass spectrometry for metabolite concentration. Results of one typical experiment are shown, $n=3$. Error bars indicate standard deviation. 


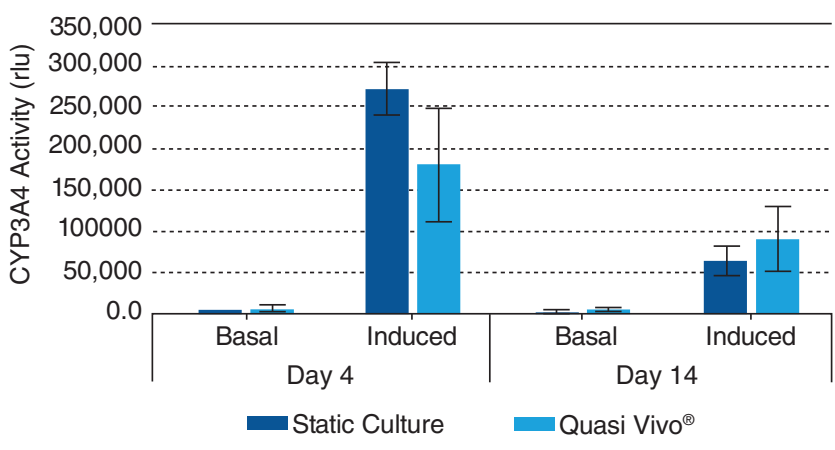

FIG. 5. PHH cultured in both static and flow conditions are responsive to CYP3A4 induction. $\mathrm{PHH}$ in collagen/Matrigel sandwich culture were cultured for the indicated period of time in either static conditions or in QV900 Flow System. Seventytwo hours before analysis, cells were stimulated with $10 \mu \mathrm{M}$ rifampicin or DMSO control before measuring CYP3A4 activity with the luminescent P450-Glo CYP3A4 Assay (Promega). Results of one typical experiment are shown, $n=3$. Error bars indicate standard deviation. DMSO, dimethyl sulfoxide.

potential drug-drug interaction. To ensure that $\mathrm{PHH}$ cultured in flow conditions still respond to specific compounds by the upregulation of CYP activity, we treated those cultures for 72 hours with rifampicin. This resulted in comparable CYP3A4 induction in both perfused and nonperfused cultures. For example, an $\sim 27$-fold increase in CYP3A4 activity was observed in the QV900 System at day 4 and $\sim 18$-fold increase at day 14 as assessed by the luciferase assay (Fig. 5).

\section{Discussion}

Fluid shear stress is known to influence the viability and functionality of hepatocytes. ${ }^{16-19}$ The Quasi Vivo System is functionally designed to apply low-level shear stress to cells cultured in monolayers or on scaffolds using a common peristaltic pump. Previous results showed that hepatocytes cultured using a low shear stress generic bioreactor system retained the metabolic activity over 3 weeks in culture. ${ }^{19}$ The chambers made of a silicone material enables better oxygen permeability for the closed culture system, but conversely causes many chemicals to become irreversibly bound to the silicone, and therefore are not ideal for longerterm toxicity studies. ${ }^{21-23}$ The QV900 is made from an acrylate plastic to reduce nonspecific binding. ${ }^{20}$ Conversely, the acrylate plastic is less permeable to oxygen ${ }^{24}$; therefore functional equivalence for hepatocytes could not be assumed. Furthermore, previous studies used only freshly isolated hepatocytes. The majority of hepatocyte use is currently performed using cryopreserved hepatocytes. It was unknown whether the metabolic activity could therefore be improved for previously cryopreserved cells to the same degree as freshly isolated hepatocytes.

Our results show that cryopreserved $\mathrm{PHH}$ can be cultured in the QV900 Flow System and improved basal CYP3A4, CYP1A2, and CYP2B6 activity is observed in perfused cultures. These results align well with a previous study showing increased cytochrome mRNA expression in flow culture. ${ }^{19}$ While the increase in metabolic enzyme activity over time in perfused conditions seems to be surprising on a first glance, it could maybe be explained by a recovery of hepatocyte functionality over time. It can be expected that cryopreserved primary cells are stressed not just by the isolation, but additionally, the cryopreservation, thawing, and seeding process. Our results show that some recovery of metabolic activity in cryopreserved hepatocytes was steady over the 3 weeks of observation. Due to the destructive nature of the experimental testing, the perfusion culture was not maintained longer than 3 weeks.

One of the many drawbacks of using PHH for long-term toxicity studies is the need for frequent medium changes. In standard static culture, daily medium changes impose rapid changes in nutrient and growth factor concentrations onto the cells and are therefore an additional source of stress for cultured cells. In perfusion culture, 50\% of the medium in the QV900 System was changed only twice a week. After each medium change, the actual medium composition in the respective wells changed only slowly. With a flow rate of $130 \mu \mathrm{L} / \mathrm{min}$ and an overall medium volume of $4 \mathrm{~mL}$ per well, it would take at least 30 minutes for a complete medium change. Due to the gradual mixing of old and new medium, this process is effectively even slower. Therefore, it is likely that the recovery of CYP450 activities observed is, in part, due to conditioning of the medium with native hepatocyte growth factors that are allowed to accumulate in the medium system. Furthermore, for application to toxicity models, reducing the frequency of necessary medium changes enables the buildup of toxic metabolites that may better mimic mechanisms of longer-term delayed-onset toxicity of drugs in the clinic. Along this line, one can envision more complex models that incorporate co-cultures of hepatocytes with other liver cell types (such as Kupffers, Stellates, and liver sinusoidal endothelial cells) in Quasi Vivo System, enabling broad applicability to long-term toxicity studies.

A major benefit of the Quasi Vivo system was the ease of setup and use. The design of the QV900 System allowed the culture of cells on standard 24-well glass coverslides. These coverslides can be easily transferred into the system and back into standard cell culture plates to allow analysis with established methods. In addition, such coverslides can be used directly for immunocytochemical staining followed by microscopic analysis. Therefore, the system can be established and analyzed using standard cell culture analysis methods.

\section{Conclusion}

In summary, we present a cell culture flow system that facilitates the long-term culture of $\mathrm{PHH}$ and restores/improves longer-term stability of drug metabolism enzymes in vitro. Therefore, the presented system can serve as an advanced human in vitro liver model, particularly suitable for longterm repeat dosing liver toxicity studies.

\section{Author Disclosure Statement}

All authors are Lonza employees. Lonza is the provider of Primary Human Hepatocytes and HCM $^{\mathrm{TM}}$ Medium used in this study and the worldwide distributor of the Quasi Vivo ${ }^{\circledR}$ System.

\section{References}

1. La Rochelle P, Lexchin J, Simonyan D. Analysis of the drugs withdrawn from the US market from 1976 to 2010 for safety reasons. Pharm Med 2016:30;277-289.

2. Olson H, Betton G, Robinson D, et al. Concordance of the toxicity of pharmaceuticals in humans and in animals. Regul Toxicol Pharmacol 2000:32;56-67. 
3. Martignoni M, Groothuis GM, de Kanter R. Species differences between mouse, rat, dog, monkey and human CYP mediated drug metabolism, inhibition and induction. Expert Opin Drug Metab Toxicol 2006:2;875-894.

4. National Research Council. Toxicity Testing in the 21st Century: A Vision and a Strategy. Washington DC: National Academy Press; 2007.

5. Godoy P, Hewitt NJ, Albrecht U, et al. Recent advances in 2D and $3 \mathrm{D}$ in vitro systems using primary hepatocytes, alternative hepatocyte sources and non-parenchymal liver cells and their use in investigating mechanisms of hepatotoxicity, cell signaling and ADME. Arch Toxicol 2013:87;1315-1530.

6. Gómez-Lechón MJ, Tolosa L, Conde I, et al. Competency of different cell models to predict human hepatotoxic drugs. Expert Opin Drug Metab Toxicol 2014:10;1553-1568.

7. Rowe C, Gerrard DT, Jenkins R, et al. Proteome-wide analyses of human hepatocytes during differentiation and dedifferentiation. Hepatology 2013:58;799-809.

8. Azimifar SB, Nagaraj N, Cox J, et al. Cell-type-resolved quantitative proteomics of murine liver. Cell Metab 2014: 20;1076-1087.

9. Lauschke VM, Vorrink SU, Moro SM, et al. Massive rearrangements of cellular MicroRNA signatures are key drivers of hepatocyte dedifferentiation. Hepatology 2016:64;1743-1756.

10. Dunn JC, Tompkins RG, Yarmush ML. Long-term in vitro function of adult hepatocytes in a collagen sandwich configuration. Biotechnol Prog 1991:7;237-245.

11. Dunn JC, Yarmush ML, Koebe HG, et al. Hepatocyte function and extracellular matrix geometry: Long-term culture in a sandwich configuration. FASEB J 1989:3;174-177.

12. Kern A, Bader A, Pichlmayr R, et al. Drug metabolism in hepatocyte sandwich cultures of rats and humans. Biochem Pharmacol 1997:54;761-772.

13. LeCluyse EL, Audus KL, Hochman JH. Formation of extensive canalicular networks by rat hepatocytes cultured in collagen sandwich configuration. Am J Physiol 1994:266; C1764-C1774.

14. Kaplowitz N. Idiosyncratic drug hepatotoxicity. Nat Rev Drug Discov 2005:4;489-499.

15. Rawlins MD. Cutting the cost of drug development? Nat Rev Drug Discov 2004:3;360-364.
16. Park J, Li Y, Berthiaume F, et al. Radial flow hepatocyte bioreactor using stacked microfabricated grooved substrates. Biotechnol Bioeng 2008:99;455-467.

17. Tilles AW, Baskaran H, Roy P, et al. Effects of oxygenation and flow on the viability and function of rat hepatocytes cocultured in a microchannel flat-plate bioreactor. Biotechnol Bioeng 2001:73;379-389.

18. Shvartsman I, Dvir T, Harel-Adar T, et al. Perfusion cell seeding and cultivation induce the assembly of thick and functional hepatocellular tissue-like construct. Tissue Eng Part A 2009:15;751-760.

19. Vinci B, Duret C, Klieber S, et al. Modular bioreactor for primary human hepatocyte culture: Medium flow stimulates expression and activity of detoxification genes. Biotechnol J 2011:6;554-564.

20. Mukhopadhyay R. When PDMS isn't the best. What are its weaknesses, and which other polymers can researchers add to their toolboxes? Anal Chem 2007:79;3248-3253.

21. Toepke MW, Beebe DJ. PDMS absorption of small molecules and consequences in microfluidic applications. Lab Chip 2006:6;1484-1486.

22. Halldorsson S, Lucumi E, Gómez-Sjöberg R, et al. Advantages and challenges of microfluidic cell culture in polydimethylsiloxane devices. Biosens Bioelectron 2015:63;218-231.

23. van Meer BJ, de Vries H, Firth KSA et al. Small molecule absorption by PDMS in the context of drug response bioassays. Biochem Biophys Res Commun 2017:482;323-328.

24. Favre E. Polymeric membranes for gas separation. In: Comprehensive Membrane Science and Engineering Volume 1. E Drioli, L Giorno (eds); pp. 158-208. Oxford: Academic Press; 2010.

Address correspondence to: Dr. Magdalene Stosik Lonza Cologne GmbH Nattermannallee 1 Cologne D-50829 Germany

E-mail: magdalene.stosik@lonza.com 